\title{
EL PRINCIPIO DE POLIEDRICIDAD: LA ARTICULACIÓN DE LO DISCURSIVO, LO COGNITIVO Y LO LINGÜÍSTICO EN TERMINOLOGÍA (I) ${ }^{1}$
}

\author{
M. Teresa Cabré*
}

RESUMO: A Terminologia é um campo interdisciplinar que distingue distintos planos ou dimensões: un plano referencial, que compreende o âmbito dos objetos e da formação de classes de objetos; um plano cognitivo, que inclui a formação de conceitos e sua localização na mente, e um plano linguístico amplamente semiótico, que compreende a realização de cada conceito em uma ou mais unidades terminológicas da língua natural, que podem ser estendidas a outras unidades de criação artificial. Este texto apresenta, em primeiro lugar, diferentes concepções do termo ou unidade terminológica e discute sua posição central ou periférica na Terminologia como campo de conheciment; em seguida, aborda a polêmica relação entre a realidade e sua representação mental na forma de conceitos de especialidade e justifica a compexidade da relação entre conceito e termo a partir do princípio de poliedricidade.

PALAVRAS-CHAVE: terminologia, conceitos de especialidade, variação conceptual, variação denominativa, representação do conhecimento especializado

El carácter interdisciplinario de la Terminología como campo del saber raramente ha sido puesto en discusión. Con independencia de la concepción teórica en la que unos y otros nos movemos, hay un consenso explícito o implícito de que la Terminología es un campo de conocimiento cuyos fundamentos tienen su origen en otros campos del saber. En contraste con esta situación de uniformidad relativa a la interdisciplinariedad de la Terminología como campo de conocimiento, la consideración de qué se entiende por término ha tenido y sigue teniendo concepciones diferentes. La noción de unidad terminológica se percibe distintamente muchas veces en función de la perspectiva desde la que se intenta describirla y explicarla.

También ha sido objeto de polémica el tema de la ubicación de la terminología en el conglomerado de las ciencias. La terminología fue concebida en la práctica como una herramienta auxiliar de la comunicación científico-técnica, cuya finalidad era asegurar la univocidad de los intercambios comunicativos entre profesionales. Esta consideración de la terminología fue superada por la decisión de Eügen Wüster de darle el rango de disciplina de pleno derecho, aunque este estatus estuviera completamente condicionado a la perspectiva práctica en la que se situó.

Wüster, como hemos expuesto en otras ocasiones (CABRÉ, 2005), tenía una gran formación como ingeniero y unas necesidades que procedían del campo de la comunicación técnica, en un contexto internacional de transferencia plurilingüe. Para ello propuso que la meta del trabajo terminológico fuera la elaboración de recursos terminológicos normalizados, es decir, fijados previamente y voluntariamente por una comunidad experta. Este punto de partida generó una

\footnotetext{
${ }^{1}$ Este artículo, que es el primero de un proyecto más amplio denominado globalmente Poliedricidad e integración: la articulación de lo discursivo, lo cognitivo y lo lingüístico en terminología, se centra en el proceso objeto-concepto-término y aborda más específicamente la complejidad de la relación concepto término. Forma parte del Proyecto TEXTERM3: Fundamentos, estrategias y herramientas para el procesamiento, extracción y representación de información especializada (HUM2006-09458/FILO 20062009). Agradezco a la Dra. Rosa Estopà los comentarios que nos ha aportado tras una lectura de la versión previa de este texto. Texto publicado originalmente em Ibérica v. 1, n. 16. Castelló: AELFE (Asociación Europea de Lenguas para Fines Específicos European Association of Languages for Specific Purposes, 2008.

* Institut Universitari de Linguística Aplicada, Universitat Pompeu Fabra (Barcelona).
} 
concepción de la materia que se concretó en varios puntos, de los que, sin pretender ser exhaustivos, destacaremos los siguientes:

En primer lugar, se estableció el objeto inicial de la disciplina: el concepto, o mejor dicho, las estructuras de conceptos. La formación de Wüster le llevó necesariamente a una concepción onomasiológica de la terminología: como se daba por supuesto el conocimiento de la materia de especialidad, este conocimiento era el punto de partida para dar cuenta de los términos, que servían para denominar cada uno de los conceptos en los que se plasmaba este conocimiento.

En segundo lugar, el pragmatismo que persigue la normalización terminológica, que debía y debe asegurar al máximo la univocidad en la transferencia de los conocimientos técnicos, llevó a Wüster a no considerar la complejidad de la relación entre la realidad y su representación. Para él la realidad técnica no tenía doblez alguna. Era única para todos los expertos del mismo campo técnico. En consecuencia, la relación entre objeto y concepto era directa. Un concepto se concebía como "la" representación de un objeto o, mejor dicho, de una clase de objetos de la realidad.

En tercer lugar, la unidimensionalidad de la relación entre objeto y concepto, juntamente con la normalización de los términos como objetivo a cubrir, determinó necesariamente la unidimensionalidad entre concepto y término, de ahí el principio de biunivocidad expuesto por la Teoría General de la Terminología como el ideal a alcanzar.

En cuarto lugar, la relación directa y biunívoca entre término y concepto explica que el término - una vez fijado a través de la actividad normalizadora - se concibiera como una unidad de designación, y no como una unidad de significación. Un término servía para referirse a un concepto, que a su vez es la representación consensuada o normalizada de una clase de objetos de la realidad, realidad concebida de modo uniforme por la comunidad experta.

Y finalmente, una concepción restrictiva de las situaciones de comunicación especializada, limitadas a la comunicación entre profesionales, llevó a Wüster a reducir los escenarios de transferencia de conocimiento, simplificando artificialmente la complejidad de la comunicación especializada. El discurso científico-técnico se vio así como un discurso plano e idéntico en cuanto a los contenidos que se transmitían y a las formas de transmisión de estos contenidos.

En este artículo nos proponemos dos objetivos generales:

El primer objetivo es argumentar que la terminología, como campo interdisciplinario, requiere para la explicación de determinados fenómenos distinguir entre distintos planos o dimensiones. Esta distinción permite, por un lado, dar una respuesta plausible a su interdisciplinariedad, y, por otro, legitimar las distintas perspectivas que han dado lugar a concepciones teóricas aparentemente enfrentadas.

El segundo objetivo se propone mostrar las correlaciones entre estos distintos planos, correlaciones que justifican la interdisciplinariedad de la unidad terminológica en particular y permiten explicar los mecanismos que se han puesto en práctica para identificar los términos en la práctica terminográfica.

Con la vista puesta en estos dos objetivos, abordaremos tres cuestiones sobre la terminología como campo y como objeto, todas ellas relacionadas con la necesidad de distinguir planos de análisis y de establecer correlaciones entre los distintos planos para poder explicar determinados fenómenos:

- En primer lugar, presentaremos la concepción diversa de la unidad terminológica y su posición central o periférica en la Terminología como campo de conocimiento.

- En segundo lugar trataremos sobre la polémica relación entre la realidad y su representación mental en forma de conceptos de especialidad.

- Y, en tercer lugar, discutiremos la complejidad de la relación entre concepto y término en una visión interdisciplinaria de la materia.

\section{LA UNIDAD TERMINOLÓGICA}


A lo largo del siglo XX la concepción de término o unidad terminológica ${ }^{2}$ ha sido diversa. Las distintas posiciones sobre el tema pueden sintetizarse básicamente en dos:

La consideración del término únicamente como forma.

La consideración del término como signo, es decir como unidad de forma y contenido.

Situándonos en la segunda concepción, consideramos que el término es una unidad de forma y contenido indisociables entre sí que representan, en el plano de la verbalización o expresión, un concepto.

Aunque pudiera parecer de entrada que la consideración del término como signo dotado de contenido y forma es una asunción exclusiva de la lingüística y, en cambio, su consideración de mera forma corresponde a una visión desde las especialidades, ambas posiciones han sido asumidas indistintamente por especialistas en terminología de diferente procedencia disciplinar. Así, Rondeau, lingüista y sociolingüista de la Universidad de Montreal, define el término como la "forma significante de un signo de especialidad"3 (RONDEAU, 1985). Para Wüster y la mayoría de sus seguidores el término es "la forma gráfica o escrita de un signo lingüístico" (WÜSTER, 1979). Esta noción de término como forma es la que recoge hasta el año 2000 la norma ISO relativa al vocabulario del trabajo terminológico (IS0 1087-1 2000, ISO 1087-2 2000).

Lo que es cierto es que la defensa de una u otra posición tiene consecuencias de gran relieve, tanto en el plano teórico como en el aplicado.

Desde el punto de vista teórico, es evidente que si un término se concibe como una simple representación formal se están asumiendo otros dos principios:

- que se da una asociación directa entre un concepto y su representación;

- que la forma de un término y el concepto que representa se dan separadamente, de modo que el concepto es independiente de su denominación.

La primera asunción sobre la asociación directa entre un concepto y su representación implica, por un lado, una lanza a favor de la univocidad entre concepto y término defendida por Wüster ${ }^{4}$, y, por otro, la defensa del concepto como una unidad bien definida por sus características necesarias y suficientes, y siempre asociado a un ámbito temático de representación. En consecuencia, la "reaparición" de este mismo concepto - mismo porque se describe a través del mismo conjunto de características - en un ámbito distinto tendrá el estatus de nuevo término aunque la definición sea idéntica. La razón en la que se sustenta este principio es que el valor de un término se define por su relación con los demás términos que con él forman la estructura conceptual de un mismo ámbito temático. Si a pesar de mantenerse la forma y el contenido aparece el término en un ámbito diferente, este se considera una nueva unidad terminológica.

Los datos siguientes, extraídos de Tebé (2005), nos muestran cuán difícil es sostener la plausibilidad de este principio:

El término REHABILITATION puede definirse como "acción de retornar un objeto o una persona a un estado funcional que había perdido".

El banco de datos terminológicos TERMIUM, que contiene 26 registros de este término, lo asocia a 21 áreas temáticas distintas, además de atribuirle un uso general:

\footnotetext{
${ }^{2}$ Para algunos autores, hay diferencia entre término y unidad terminológica, para nosotros son dos variantes completamente sinónimas.

${ }^{3}$ Hay que decir, sin embargo, que a lo largo de su manual Rondeau vacila entre ambas denominaciones y a veces utiliza "término" con valor de signo especializado.

${ }^{4}$ Adelstein (2006) explicita que Wüster, a pesar de establecer la biunivocidad como fundamento, es totalmente consciente de que esta propiedad solo se da en las unidades ideales, pero no en las reales.
} 


Urbanismo
Ciudadanía e Inmigración
Métodos de construcción
Criminología
Ecosistema
Emergencias
Medioambiente general
Economía
medioambiental
Ocupación y trabajo
Préstamos

Fuerzas armadas

Préstamos bancarios

Administración militar

Parques y jardines

Desarrollo de páginas

web

Servicios sociales

Agricultura

Ferrocarriles

Vivienda

Renovación urbanística

Vocabulario general

La totalidad de las definiciones de rehabilitation que recoge TERMIUM comparte los rasgos básicos esenciales del concepto REHABILITATION; lo único que cambia son las proyecciones semánticas del mismo y la clase semántica de los argumentos sobre los que este término predica, argumentos que se precisan según el ámbito temático en el que se usa, además de otros aspectos no esenciales de este concepto, como son:

- la causa que produce la pérdida de habilitación

- la manera cómo se produce la rehabilitación

- el tipo de estado que se debe recuperar mediante la rehabilitación

En consecuencia, con la presentación de este ejemplo, que de hecho la lingüística clásica trataría como un fenómeno de polisemia, podríamos decir que tanto un experto en arquitectura, como un médico o un ecologista, coincidirían en el concepto de REHABILITATION que tienen en la mente; en lo que diferirían es en el hecho de que este concepto se realiza lingüísticamente en condiciones pragmáticas y discursivas distintas, y es en función de cada una de ellas que se precisan las características no esenciales.

Otro ejemplo, en este caso de sinonimia, puede aportar nuevos argumentos en contra de la relación directa entre concepto y término. Para un experto, el referente que se supone que representan tanto la unidad dinero negro, como la unidad dinero blanqueado es el mismo. Se trata de dos denominaciones distintas que comparten un núcleo común (dinero) y se diferencian por la expansión del núcleo (negro, blanqueado), que se refieren a un mismo objeto. Si asumimos que se da una correspondencia directa entre objeto y concepto, ambas unidades representarían un mismo concepto, pero sin embargo ¿cómo explicarlo? Porque, aunque es cierto que un especialista en fraude monetario sabe muy bien que el hecho que hay detrás de ambas denominaciones es el mismo, no existe lingüista alguno que considere que estas dos denominaciones son sinónimas entre si, porque expresan contenidos distintos. ${ }^{5}$

¿Podría explicarse este fenómeno utilizando como base la asociación directa objetoconcepto-término de la teoría terminológica tradicional? Si la respuesta fuera afirmativa, los términos serían meras denominaciones con función designativa. Nuestra opinión es que solo con una concepción de la unidad término como signo compuesto de forma y contenido, casos como este podrían explicarse más adecuadamente. La razón parece clara: se admitiría que los términos, como todo signo, además de denominar significan en si mismos, con lo que la terminología asumiría uno de los fundamentos esenciales de la lingüística cognitiva: la motivación de los signos del lenguaje.

Regresemos de nuevo al alcance del término en la bibliografía terminológica y centrémonos ahora en la concepción de la unidad "término" como signo. Dentro de esta aproximación

\footnotetext{
${ }^{5}$ G. Leech (1990) introduce una distinción entre sinonimia fáctica y sinonimia lingüística, que resulta muy útil para dar cuenta de este fenómeno. Dos unidades son sinónimos fácticos cuando se refieren al mismo objeto de la realidad (presidente del gobierno y jefe del ejecutivo, ambas expresiones referidas a J. L. Rodríguez Zapatero), pero no son expresiones sinónimas desde el punto de vista lingüístico porque "dicen cosas distintas" del mismo objeto. Retomaremos este tema más adelante y trataremos de darle una explicación más robusta.
} 
podemos distinguir dos posiciones:

- la de quienes consideran que un término es una denominación general para todo tipo de unidad de un sistema expresivo, con lo que los símbolos y los nombres científicos se considerarían términos;

- la de hay quienes reservan esta denominación únicamente para las unidades que pertenecen a las lenguas naturales.

Para los primeros no habría diferencia en el uso de uno u otro tipo de sistema de expresión para representar los conceptos, a pesar de que las diferencias entre los signos lingüísticos y los no lingüísticos son abismales y de que para todo concepto siempre existe un signo lingüístico y, en cambio, para todo signo lingüístico no existe necesariamente un signo no lingüístico asociado.

Los que defienden que la denominación "término" debe utilizarse únicamente para referirse a las unidades que cumplen la condición de ser signos lingüísticos asumen que los términos participan de las mismas características y se someten a las mismas condiciones que las unidades del léxico de las lenguas. Su función sería pues al mismo tiempo designativa, denominativa y significativa:

- designativa por el hecho de "apuntar a" un referente,

- denominativa por el hecho de darle nombre a un concepto, y

- significativa porque en sí mismos aportan información sobre el contenido que transmiten.

Observemos cuán diferente es esta posición de la que considera que la función de un término es solo la de designar el referente y denominar el concepto.

Otro de los aspectos que separa ambas concepciones sobre la unidad "término" se refiere al tipo de significado que dicho signo vehicula. Para quienes asumen la condición meramente designativa y denominativa de los términos, el único significado que se pone en juego mediante estas unidades es el significado denotativo. Los términos poseen un contenido que coincide y se limita a lo que expresa el concepto. El alcance de la denominación es el concepto. En contraste, para quienes aceptan la condición de signo de los términos, el significado que estos poseen comprende tanto la denotación como la connotación, ya que participan de las mismas características que poseen todas las unidades del léxico de las lenguas.

Una tercera característica que permite diferenciar concepciones distintas sobre la unidad "término" concebida como signo se basa en sus coincidencias o divergencias respecto a las unidades denominadas "palabras". Cuatro han sido las posiciones que se han mantenido sobre este tema:

- la de quienes suscriben que palabras y términos son unidades de naturaleza diferente que forman parte de conjuntos distintos;

- la de quienes consideran que términos y palabras forman parte del mismo componente del léxico de las lenguas, aunque organizados como entradas distintas;

- la de quienes sostienen que el léxico, además de términos y palabras por separado, contiene un subconjunto de unidades especializadas de naturaleza "light" que están a caballo entre los términos y las palabras;

- la de quienes asumen que un término no es una unidad en si misma, sino solo un valor asociado a todas las unidades del léxico, de forma que ninguna de ellas es por sí misma un término, sino que todas ellas pueden ser términos o no serlo en función de su uso particular en un contexto comunicativo determinado.

Los partidarios de la opción que defiende que palabras y términos son unidades de naturaleza diferente que forman parte de conjuntos distintos presuponen que existen dos conjuntos léxicos diferenciados en la mente de un hablante, uno determinado por su condición de experto, y otro justificado en su condición de mero hablante. Se trataría entonces de postular que todo hablante tiene interiorizados por lo menos dos componentes léxicos, y que actualiza uno u otro en función de la situación comunicativa en la que se encuentra. Si está hablando como experto en una situación profesional, activa el componente léxico propio de la especialidad. Si se comunica al margen del contexto profesional, simplemente no activa este componente. 
Huelga decir que esta opción resultaría poco plausible a la vista de las investigaciones que se han llevado a cabo en neurolingüística, y que dejaría sin explicar por qué una misma unidad puede ser usada como término especializado y como palabra sin especialidad alguna, a no ser que recurriéramos a la reduplicación de estas unidades en la mente, lo que nos llevaría a un modelo altamente redundante.

Los mismos argumentos servirían para descartar por inadecuada una teoría que postulara que cada unidad del léxico tendría tantas entradas como sentidos especializados asociados a ámbitos temáticos tuviere. No únicamente porque sería también una propuesta enormemente redundante, sino porque además no daría cuenta de la relación existente entre unidades que, teniendo una misma forma, pueden asociarse a ámbitos temáticos distintos con un significado básicamente idéntico, pero con algunos elementos de diferenciación, como hemos mostrado a través del ejemplo rehabilitation.

En la tercera opción se sitúan algunos autores que proponen la idea de considerar que en la mente del hablante existen tres tipos de unidades: las unidades terminológicas de contenido especializado, las unidades de sentido general, y un conjunto de unidades a medio camino entre unas y otras, que corresponderían a lo que en didáctica de las lenguas de especialidad se ha denominado léxico subtécnico (VANGEHUCHTEN, 2005). Esta propuesta, aunque reconoce que existen unidades de las que no se sabe si considerarlas términos o palabras del léxico general, puede contraargumentarse de la misma manera que en los dos casos anteriores.

Nuestra propuesta intenta dar un paso más y es cualitativamente distinta. Para nosotros, términos y palabras (aquí sinónimo para referirnos a los no términos) no son en si unidades diferentes: un término no es una unidad en sí misma sino solo un valor asociado a todas las unidades del léxico, de forma que cada una de ellas no es por si misma, como hemos dicho, ni término ni palabra, sino que activa o no su valor de término en función de su uso particular en un contexto comunicativo determinado. Esta propuesta, denominada "Principio del valor terminológico", constituye uno de los pilares sobre los que se sustenta nuestro aparato teórico.

\section{REALIDAD, REPRESENTACIÓN Y CONCEPTUALIZACIÓN DEL CONOCIMIENTO ESPECIALIZADO}

Trataremos ahora de analizar el proceso que presuntamente realiza un hablante desde la percepción de la realidad hasta su verbalización. De ningún modo pretendemos entrar en el tema desde postulados neuropsicológicos, sino solamente establecer un marco en el que enfocar los dos puntos esenciales de este texto: la necesidad de distinguir planos distintos de análisis para dar cuenta con precisión de las unidades terminológicas y su ubicación en el proceso de representación y transmisión del conocimiento especializado, y la de explicar algunas de las relaciones que se establecen entre estos planos.

No entramos tampoco en el tema de las identidades y diferencias entre los procesos de adquisición de conocimiento general en contraste o no con el especializado, sino que nos limitamos al proceso de adquisición de conocimiento especializado. Para ello, partimos del supuesto que los mecanismos de percepción de la realidad y de construcción de clases de objetos en las especialidades no son distintos de los que sirven para explicar el proceso de adquisición del conocimiento en general. La secuencia de actividades que podrían describir este proceso dependen, en nuestra opinión, de los escenarios de adquisición de conocimiento. Tomaremos como punto de partida solo dos de estos escenarios:

- un primer escenario de producción de conocimiento nuevo (ESC1);

- un segundo escenario de adquisición de conocimiento producido anteriormente, a través de la recepción de información vía discurso (ESC2).

Como se ha descrito ampliamente en las ciencias cognitivas, la segmentación del contínuum de la realidad depende de mecanismos psicocognitivos muy complejos que no son ajenos a los valores culturales interiorizados por los hablantes de toda comunidad. Aunque no tratamos de entrar a fondo en este punto, vamos a presentar muy someramente algunas fases del proceso a fin de entender posteriormente cómo la categorización de la realidad conduce a la formación de 
un concepto de estructura compleja, lo que permite explicar el comportamiento y la variación en terminología.

Se sabe que los seres humanos "perciben" la realidad a través de filtros interiorizados. Estos filtros, de carácter psicológico, antropológico y sociológico, actúan de mediadores entre una supuesta "realidad por encima de los individuos" y la realidad percibida por los seres humanos que pertenecen a comunidades y grupos determinados y se ubican en contextos históricos y sociales. La realidad pura, pues, está fuera de nuestra comprensión como seres sociales.

Si asumimos este punto de partida, podemos avanzar en el proceso situándonos en una realidad percibida socioculturalmente y discriminada psicológicamente en forma de "clases objetos", es decir, conjuntos de objetos individuales que se han agrupado en una clase por el hecho de compartir determinadas características que actúan como catalizadoras en función de su centralidad en cada grupo humano.

No vamos a discutir aquí si esta centralidad en los criterios de discriminación y agrupación está o no mediatizada por las lenguas. Nos quedaremos únicamente en la asunción de que está mediatizada por la cultura interiorizada que posee un individuo hablante, que forma parte de colectivos sociales.

Basta observar individuos que pertenecen a grupos culturales muy alejados para constatar que su "percepción", en términos de agrupación de fragmentos de realidad en una unidad o clase de objeto, no coincide. El principio básico en el que se apoya esta idea es que a contextos distintos les corresponden necesidades distintas y que las necesidades empujan a los seres humanos a "ver" fenómenos que otros individuos en condiciones distintas ni siquiera perciben. De este principio se han dado muchos ejemplos, uno de los más conocidos es el de la percepción de más de cuarenta matices de blanco por parte de una comunidad esquimal. Y es este mismo principio el que nos sirve para explicar la percepción desde las especialidades.

Volviendo a los dos tipos de contexto seleccionados más arriba y centrándonos en la adquisición de conocimiento especializado, podemos decir que en el primer escenario de producción de conocimiento nuevo (ESC1), lo que el individuo percibe, discrimina y clasifica está condicionado por sus conocimientos previos y por sus necesidades, en el marco del rol que se atribuye el individuo en cada proceso. Precisando un poco más, en los escenarios en los que un individuo, cuyo rol es el de un especialista en la materia, se acerca a la realidad lo hace desde una competencia previa (conocimientos adquiridos anteriormente desde este rol) y movido por la necesidad de descubrir nuevos fenómenos o de percibir nuevas características o relaciones de fenómenos ya anteriormente percibidos, que le permiten avanzar en su conocimiento.

En un contexto de "descubrimiento" los individuos "perciben" por primera vez un fenómeno nuevo o una propiedad nueva de un fenómeno ya observado, o establecen una nueva relación entre dos fenómenos; es decir, perciben una nueva parcela del contínuum de la realidad sobre la base de la observación de los datos empíricos. Esta observación les conduce a una constatación de que se trata de algo nuevo; y así, en el largo proceso de observación y análisis, van caracterizando y estabilizando dicho conocimiento a través de operaciones cognitivas consistentes en la abstracción y jerarquización de las características esenciales y periféricas y usando a menudo el discurso como herramienta de progresión. Una vez estabilizada la discriminación de la nueva unidad de conocimiento, se categoriza en la mente como un nuevo concepto asociado a este fragmento de realidad percibido.

Observemos que hasta ahora hemos establecido dos variables esenciales. La primera variable es el esquema o marco situacional del que el individuo parte, que incluye sus condiciones inherentes (sexo, edad, época, grupo social, grupo profesional, especialidad, escuela de pensamiento, nivel de competencia en el tema, función social, etc.), lo que determina su rol en este proceso de adquisición de conocimiento en forma de activación de filtros de adquisición. La segunda variable la constituyen los mecanismos psicocognitivos que explican cómo un individuo dentro de un marco situacional es capaz de crear unidades de conocimiento en forma de conceptos a partir de una realidad que es un contínuum.

El proceso que se produce en el segundo escenario, que hemos caracterizado como de

${ }^{6}$ Hablamos de "descubrimiento" sólo en sentido metafórico. 
adquisición de conocimiento producido anteriormente a través de la recepción de información vía discurso (ESC2), es bastante distinto del anterior. En este caso no se trata de la adquisición de conocimiento nuevo a partir de la observación de la realidad, sino de la adquisición de conocimiento ya estabilizado, expresado discursivamente. Lo que el individuo activa en este contexto son los mecanismos que le permiten "detectar" las unidades de conocimiento expresadas en el texto oral o escrito ya formulado. En este proceso de detección hay que tener en cuenta las condiciones previas inherentes de quien lo lleva a cabo, sus intenciones y las finalidades para las que lo lleva a cabo. Todo este conjunto de condiciones las hemos definido en el escenario anterior como el marco en que el individuo se sitúa, que, como también hemos visto en el primer escenario, mediatiza sus posibilidades y actividades. Lo que diferencia claramente este segundo escenario del anterior es que el individuo no "construye" un nuevo conocimiento a través de la cognición y el discurso, sino que trata de detectar unidades de conocimiento contenidas como tales en un texto. Se basa pues en el análisis del texto, y no en el análisis de la realidad, para adquirir conocimiento. Trata de "descubrir" las unidades de conocimiento que presumiblemente "están" en el texto. Se trata pues de un proceso claramente semasiológico, de un mecanismo de adquisición de conocimiento a partir de un proceso de descodificación mediatizado.

Este nuevo conocimiento se ubica en la mente humana en forma de unidades conceptuales más o menos complejas. El proceso y la forma de ubicación son temas que escapan a nuestra propuesta, pero de los que podemos decir que están condicionados por las hipótesis psicocognitivas o neurocognitivas de las que se parte. Desde el punto de vista neurocognitivo se ha lanzado dos hipótesis: la primera afirma que cada tipo de conocimiento está localizado en un lugar de la mente, la segunda propone que el conocimiento no se agrupa por tipos sino que se encuentra distribuido en la mente.

Al lado del interrogante sobre la ubicación mental del conocimiento, existe un segundo problema: ¿cómo se estructura este conocimiento?: ¿se trata de una estructuración jerárquica o de un modelo organizado en red? Tampoco es nuestro objetivo dar una respuesta a estos interrogantes, aunque por lo que diremos a continuación se observe que nos decantamos más por la organización de los conceptos en una estructura de red, ${ }^{7}$ sino únicamente dar cuenta de que la información extraída de la realidad en las condiciones que hemos descrito, y ubicada como clase o categoría en la mente, establece relaciones con el resto de categorías también interiorizadas, y que estas relaciones no son bidimensionales sino multidimensionales, de forma que un concepto interiorizado establece relaciones de mayor o menor intensidad o de mayor o menor cercanía con la totalidad de los conceptos de la mente.

Uno de los puntos que han inquietado y sigue inquietando a quienes se ocupan del conocimiento es cómo se describen los conceptos. Es evidente que desde nuestra posición científica no podemos abordar los conceptos directamente y extraer conclusiones que sean empíricamente adecuadas, pero lo que sí queremos plantear es cómo a través de la observación de las unidades terminológicas que representan conceptos en el discurso especializado se pueden hacer inferencias sobre las características que presumiblemente poseen y las condiciones que cumplen.

Pongamos pues a la vista algunos de los datos que pueden guiar nuestro análisis del concepto:

Sabemos que en el discurso un término lo es porque tiene un sentido preciso en un ámbito de conocimiento determinado y es usado significativamente en el discurso especializado de este ámbito.

- Sabemos también que este término se corresponde a un concepto en la mente del especialista en el tema, porque cuando usa un término lo asocia a una unidad conceptual precisa dentro de su ámbito de especialidad.

\footnotetext{
${ }^{7}$ Solo un modelo en red permite, a nuestro juicio, explicar la movilidad de los términos entre ámbitos temáticos. Aceptar esta dinámica es coherente con el principio que hemos expuesto en otros artículos según el cual los términos no "pertenecen" a un ámbito sino que se usan en uno o más ámbitos. Esta afirmación no excluye que podamos decir que un término ha aparecido por primera vez en un ámbito.
} 
- Sabemos además que alguien es especialista cuando conoce una especialidad, y que conocer una especialidad presupone tener una estructura conceptual general de cada saber en la mente, reconocida, aunque con posibles variantes o alternativas, por la comunidad experta, de forma que el individuo considerado especialista es capaz, por un lado, de expresar conocimiento sobre la materia de acuerdo con esta estructura variada aunque estabilizada, y, por otro lado, reconocer discursos especializados no solo por su expresión y formato, sino sobre todo por su contenido. Asimismo es capaz de detectar transgresiones conceptuales que un no experto nunca percibiría.

- También sabemos que existe variación terminológica para representar un mismo concepto, lo que se conoce genéricamente como sinonimia. Esta variación no solo la reconocen los especialistas sino que son ellos mismos quienes la producen.

- Sabemos que una misma unidad de conocimiento puede aparecer en las producciones de los especialistas no solo denominada de manera diferente (sinonimia) sino también "explicada" o expuesta discursivamente de manera diferente, ya sea en bloque, ya sea introduciendo progresivamente aspectos distintos de la unidad conceptual.

- Finalmente sabemos que una unidad formal puede usarse asociada a más de un sentido, dentro de un mismo ámbito temático o en distintos ámbitos temáticos, lo que se conoce genéricamente como polisemia u homonimia en función de la posibilidad o imposibilidad de explicar la variación semántica desde una sola unidad. ${ }^{8}$

- Sintetizando los datos anteriores podemos decir:

- que un concepto es una unidad que forma parte de una estructura más compleja, y que esta estructura corresponde a la representación de cómo un individuo o grupo concibe un área de conocimiento;

- que un concepto representa una parcela "discriminada" a partir de una observación experiencial, mediatizada por filtros, y "construida" por comparación a partir de la observación, la discriminación y el análisis de la realidad y tomando como punto de referencia categorías idealizadas en la mente;

- que esta construcción corresponde a un proceso de categorización o conceptualización (formación de un concepto) que conduce a la ubicación de esta categoría en la mente, en el caso que nos ocupa, de un especialista;

- que un concepto, por lo tanto, es el resultado de la categorización en la mente de lo que se ha "construido" como una clase de objeto y no como un objeto particular;

- que un concepto mental interiorizado puede formar parte de más de una estructura conceptual si tenemos en cuenta que, en forma de término, aparece en discursos de distinto tema con las mismas características básicas, lo que nos llevaría a poner sobre la mesa el carácter "virtual" y no "real" de las estructuras conceptuales;

- que un concepto, además de poder aparecer en distintos ámbitos temáticos con el mismo contenido básico (reutilización de un concepto), algunas veces lo hace con algunas características periféricas o complementarias diferentes, que determinan casi siempre combinaciones léxicas distintas en el discurso, pero que pueden explicarse a partir de un esquema de características común;

- que un concepto puede ser representado en el discurso por más de una unidad de expresión, lo que se conoce con el término de sinonimia;

- que un mismo concepto, categorizado a partir de un objeto, puede describirse como una estructura compleja por el hecho de que cuando se verbaliza en unidades distintas cada una de estas unidades puede remitirnos a aspectos diferentes de este concepto.

Nos quedaremos pues por el momento en que las relaciones entre realidad, objeto, concepto y término que podemos observar indirectamente a partir de datos del discurso serían las siguientes:

\footnotetext{
${ }^{8}$ En la lingüística más ortodoxa, la homonimia se reserva para las unidades que proceden de formas etimológicas distintas. No serían homónimas por tanto las unidades cuyo sentido se hubiera generado por restricción, ampliación o cambio de un significado de base.
} 


\begin{tabular}{|l|l|l|l|}
\hline $\begin{array}{l}\text { Plano de los objetos } \\
\text { individuales }\end{array}$ & $\begin{array}{l}\text { Plano de las clases de } \\
\text { objetos }\end{array}$ & Plano conceptual & Plano terminológico \\
\hline $\begin{array}{l}\text { conjunto de objetos } \\
\text { individuales }\end{array}$ & un objeto discriminado & un concepto & un término \\
\hline $\begin{array}{l}\text { conjunto de objetos } \\
\text { individuales }\end{array}$ & $\begin{array}{l}\text { varios objetos } \\
\text { discriminados }\end{array}$ & varios conceptos & $\begin{array}{l}\text { varios términos distintos } \\
\text { o formalmente iguales } \\
\text { (homonimia) }\end{array}$ \\
\hline $\begin{array}{l}\text { conjunto de objetos } \\
\text { individuales }\end{array}$ & un objeto discriminado & un concepto & $\begin{array}{l}\text { varios términos } \\
\text { (sinónimos) sin } \\
\text { consecuencias cognitivas }\end{array}$ \\
\hline $\begin{array}{l}\text { conjunto de objetos } \\
\text { individuales }\end{array}$ & un objeto discriminado & un concepto & $\begin{array}{l}\text { varios términos } \\
\text { (sinónimos) con } \\
\text { consecuencias cognitivas }\end{array}$ \\
\hline
\end{tabular}

Fig. 1 Relaciones entre realidad, objeto, concepto y término.

Veamos un ejemplo de cada caso, limitándonos en este apartado a las relaciones entre el plano de los objetos y el plano conceptual, y dejando para el apartado siguiente su complementación con el plano de los términos:

\begin{tabular}{|l|l|l|}
\hline $\begin{array}{l}\text { Plano de los objetos } \\
\text { individuales }\end{array}$ & $\begin{array}{l}\text { Plano de las clases } \\
\text { de objetos }\end{array}$ & \multicolumn{1}{|c|}{ Plano conceptual } \\
\hline $\begin{array}{l}\text { conjunto de objetos } \\
\text { individuales }\end{array}$ & $\begin{array}{l}\text { asociación a una } \\
\text { sola categoría }\end{array}$ & $\begin{array}{l}\text { un concepto } \\
\text { "seno del complemento de un ángulo o de un arco" }\end{array}$ \\
\hline $\begin{array}{l}\text { conjunto de objetos } \\
\text { individuales }\end{array}$ & $\begin{array}{l}\text { asociación a } \\
\text { distintas categorías }\end{array}$ & $\begin{array}{l}\text { varios conceptos } \\
\text { "signo ortográfico que indica una pausa breve" } \\
\text { "estado patológico que se caracteriza por la pérdida de } \\
\text { la conciencia, la sensibilidad y la capacidad motora } \\
\text { voluntaria" }\end{array}$ \\
\hline $\begin{array}{l}\text { conjunto de objetos } \\
\text { individuales }\end{array}$ & $\begin{array}{l}\text { un objeto } \\
\text { discriminado }\end{array}$ & $\begin{array}{l}\text { un concepto que se proyectará en el próximo cuadro en } \\
\text { "afección cutánea caracterizada por vesículas rojizas y } \\
\text { exudativas, que dan lugar a costras y escamas" }\end{array}$ \\
\hline $\begin{array}{l}\text { conjunto de objetos } \\
\text { individuales }\end{array}$ & $\begin{array}{l}\text { un objeto } \\
\text { discriminado }\end{array}$ & $\begin{array}{l}\text { uncepton que en el próximo cuadro se desglosará en } \\
\text { "difusión mundidos de modos, valores o tendencias que } \\
\text { fomenta la uniformidad de gustos y costumbres" }\end{array}$ \\
\hline
\end{tabular}

Fig. 2 Relaciones entre el plano de los objetos y el plano conceptual.

Este cuadro desea representar las posibles interrelaciones entre tres planos: el de los objetos individuales que hipotéticamente se encuentran en la realidad "real" (valga la redundancia), el de las clases de objetos que se integran en una realidad abstracta, y el de los conceptos. El paso del primero al segundo plano se explica por mecanismos de discriminación, análisis y comparación, de forma que se pasa de los objetos individuales a las clases de objetos. El paso del segundo al tercer plano se basa en un proceso de categorización, y permite explicar el paso de la clase de objetos a conceptos, que se organizan internamente como esquemas de rasgos en estructuras de naturaleza prototípica, y que pueden proyectarse multidimensionalmente, como veremos más adelante.

En todos los casos, intentamos representar la construcción de una clase de objeto y su proyección en una categoría conceptual, y, aunque en el segundo se parte de más de un objeto proyectado en más de un concepto, no se trata de ninguna excepción, sino únicamente de la 
repetición del mismo proceso.

\section{LAS RELACIONES CONCEPTO Y TÉRMINO}

Analizaremos en este apartado las complejas relaciones entre conceptos y términos. Para dar cuenta de ellas empezaremos hablando de los conceptos, de sus características y de su representación, y a continuación retomaremos la concepción de unidad terminológica como signo, en la línea de lo que se ha expuesto en el apartado 1 de este artículo.

Tanto las publicaciones de Wüster como las normas ISO explican los conceptos como conjuntos de características (denominadas también propiedades) de índole diversa: "Unit of thought constituted through abstraction on the basis of properties of a set or one or more objects." (ISO-1987-1 1997).

Esta concepción se ha modificado en la norma del 2000 como: "Unit of knowledge created by a unique combination of characteristics." (ISO-1987-1 2000).

Muchas han sido las aportaciones que desde las ciencias cognitivas se han hecho sobre la conceptualización o formación de conceptos. Entre ellas merece destacarse la teoría del prototipo formulada por E. Rosch en 1978 y difundida en lingüística gracias a la obra de G. Lakoff de 1987. De acuerdo con esta propuesta, la formación de categorías no se lleva a cabo, como se había sostenido, sobre la base de la comparación de las características de distintos objetos existentes en la realidad con el objeto de extraer qué características son comunes a todos ellos o a parte de ellos, sino que se realiza sobre la base de cómo el individuo percibe la realidad y comparando el conjunto de características de los objetos en referencia a un modelo ideal cognitivo a través de cuatro mecanismos comunes a la especie humana: la estructura en proposiciones, la estructura en imágenes, la proyección metafórica y la proyección metonímica. Es sobre esta base que Temmerman (2000) se sirve del término understanding unit para referirse al resultado del proceso de categorización.

Las categorías, en esta aproximación, no se conciben como clases cerradas ni de límites totalmente establecidos, sino como estructuras organizadas internamente e intercategorialmente. La estructuración interna de las categorías responde al modelo del prototipo, con un núcleo y una periferia, que permite reconocer los mejores ejemplares de cada tipo según se correspondan al modelo. Las distintas categorías se estructuran en tres niveles distintos de categorización: el nivel básico, que es el nivel natural de categorización, el nivel superordinado que permite agrupar categorías del nivel básico en categorías más complejas, y el nivel subordinado que permite agrupar las categorías a partir de la comparación de sus atributos.

En consecuencia con estas ideas, un concepto no puede definirse como una estructura cerrada de rasgos definitorios, sino como una categoría que se enmarca en un esquema de rasgos que la definen. Es en referencia a este esquema que se compara cognitivamente cada ejemplar de la realidad con el ideal, para poder determinar si se trata o no de un ejemplar de esta categoría o bien no puede ser incluido en ella.

Pero no queremos tratar en este artículo de cómo los distintos casos se van asociando a categorías, sino que nuestro propósito es, por un lado, analizar las relaciones que se pueden establecer entre unidades de categoría conceptual y unidades terminológicas y así poder explicarnos cómo un concepto puede representarse en el plano lingüístico a través de más de un término. Y en segundo lugar, vamos a intentar iniciar la reflexión sobre las diferencias entre lo que se denomina variación propiamente conceptual y la variación denominativa con consecuencias conceptuales.

Retomamos a continuación el cuadro del apartado anterior y lo complementamos con un nuevo plano, el plano terminológico:

\begin{tabular}{|l|l|l|l|}
\hline $\begin{array}{l}\text { Plano de los objetos } \\
\text { individuales }\end{array}$ & $\begin{array}{l}\text { Plano de las clases de } \\
\text { objetos }\end{array}$ & Plano conceptual & Plano terminológico \\
\hline conjunto de objetos & un objeto discriminado & un concepto "seno del & un término \\
\hline
\end{tabular}




\begin{tabular}{|c|c|c|c|}
\hline individuales & & $\begin{array}{l}\text { complemento de un } \\
\text { ángulo o de un arco" }\end{array}$ & coseno \\
\hline $\begin{array}{l}\text { conjunto de objetos } \\
\text { individuales }\end{array}$ & $\begin{array}{l}\text { varios objetos } \\
\text { discriminados }\end{array}$ & $\begin{array}{l}\text { varios conceptos } \\
\text { "estado patológico que } \\
\text { se caracteriza por la } \\
\text { pérdida de la } \\
\text { conciencia, la } \\
\text { sensibilidad y la } \\
\text { capacidad motora } \\
\text { voluntaria" "signo } \\
\text { ortográfico que indica } \\
\text { una pausa breve" }\end{array}$ & $\begin{array}{l}\text { varios términos } \\
\text { distintos o formalmente } \\
\text { iguales (homonimia): } \\
\text { coma (signo } \\
\text { ortográfico) } \\
\text { coma (estado } \\
\text { patológico) }\end{array}$ \\
\hline $\begin{array}{l}\text { conjunto de objetos } \\
\text { individuales }\end{array}$ & un objeto discriminado & $\begin{array}{l}\text { un concepto "afección } \\
\text { cutánea caracterizada } \\
\text { por vesículas rojizas y } \\
\text { exudativas, que dan } \\
\text { lugar a costras y } \\
\text { escamas" }\end{array}$ & $\begin{array}{l}\text { varios términos } \\
\text { (sinónimos) sin } \\
\text { consecuencias } \\
\text { cognitivas } \\
\qquad \begin{array}{c}\text { eccema } \\
\text { eczema } \\
\text { erupción }\end{array} \\
\end{array}$ \\
\hline $\begin{array}{l}\text { conjunto de objetos } \\
\text { individuales }\end{array}$ & un objeto discriminado & $\begin{array}{l}\text { un concepto "difusión } \\
\text { mundial de modos, } \\
\text { valores o tendencias que } \\
\text { fomenta la uniformidad } \\
\text { de gustos y costumbres" }\end{array}$ & $\begin{array}{l}\text { varios términos } \\
\text { (sinónimos) con } \\
\text { consecuencias } \\
\text { cognitivas } \\
\text { mundialización } \\
\text { globalización }\end{array}$ \\
\hline
\end{tabular}

Fig. 3 Relaciones entre el plano de los objetos, el plano conceptual y el plano terminológico.

El primer tipo de caso es muy poco frecuente: el término coseno es un término puramente matemático cuyo significado representa un concepto en su globalidad y corresponde a un objeto "construido" desde la realidad en un esquema determinado (el de la Matemática).

El segundo caso no merece más atención que decir que representa la totalidad de los casos de homonimia, es decir, de realidades verdaderamente distintas y no relacionadas entre sí, que han generado conceptos claramente distintos cada uno de los cuales sí que se representa mediante un término, presentando estos distintos términos una coincidencia formal entre si. La única relación que guardan estos casos es en el plano del término y aun únicamente por el hecho de que haya una coincidencia formal, y solo formal, entre las denominaciones.

Los casos que merecen más atención son los dos últimos. ${ }^{* *}$ Ambos intentan ejemplificar cómo un objeto puede representarse verbalmente a través de distintos términos. ${ }^{9}$ En esta relación pueden darse dos casos:

a) que este objeto corresponda a un solo concepto, expresado por diferentes signos semánticamente coincidentes, aunque formalmente diferentes (caso ${ }^{\circ} 3$ ),

b) que este objeto corresponda a un solo concepto, expresado por diferentes signos semánticamente y formalmente diferentes (caso $\mathrm{n}^{\circ} 4$ ).

En el caso número 3 pueden incluirse ejemplos de índole distinta: por una parte variantes puramente gráficas (eczema-eccema), por otra, variantes morfológicas (alborotoalborotamiento; condiciones climáticas-condiciones climatológicas) y en tercer lugar variantes léxicas (bencina-esencia-gasolina). La característica común que comparten es el hecho de que usar una u otra variante no cambia la manera cómo se representa el concepto, o dicho de otro modo, la manera cómo el concepto se proyecta en la denominación. En todos los casos tan opaca o transparente es una variante como otra, y, desde el punto de vista cognitivo, el uso de una u otra variante no introduce cambios en la proyección del concepto en el discurso ni

\footnotetext{
${ }^{9}$ Queremos recordar que en este trabajo consideramos término o unidad terminológica aquella unidad sígnica dotada de forma (que corresponde a la denominación) y contenido (que corresponde al significado).
} 
presupone tampoco una intención diferente (a no ser que sea estilística o extralingüística) por parte del locutor.

Muy distinto es en cambio el caso número 4, que podría complementarse con otros ejemplos como:

agricultura ecológica-agricultura biológica

mundialización-globalización

residuos biosanitarios-residuos hospitalarios

contaminación del aire-contaminación atmosférica

En todos estos ejemplos podemos ver que el uso de una variante u otra para designar un mismo objeto podría estar condicionado por una intención cognitiva por parte del locutor y, evidentemente, tiene consecuencias cognitivas en el receptor. Analicemos más detenidamente uno de los casos: residuos biosanitarios-residuos hospitalarios, tomado de Freixa (2002).

Es evidente que el objeto designado por los dos términos en relación de variación es el mismo: "los deshechos que se producen por actividades clínicas relacionadas con la vida y la salud". Estos residuos se producen fundamentalmente en los hospitales, donde se ejercen actividades "clínicas relacionadas con la vida y la salud". Pero existen dos denominaciones y cada una de ellas nos remite por si misma a un sentido diferente: en el primer caso la denominación se centra en las características de las actividades que provocan este tipo de residuos (actividades biosanitarias); en el segundo, en el ámbito en el que se producen estos residuos (hospitales). No hay duda de que se trata de dos signos formados sintagmáticamente a partir de un núcleo común (residuos) y cuyo significado coincide parcialmente. Lo que se trata de saber, sin embargo, es si esta sinonimia parcial puede explicarse a partir de un único concepto o si cada sentido de un término remite a un concepto distinto aunque haya tantos puntos en común entre ambos sentidos.

Retomando ahora la concepción de la unidad terminológica como un signo compuesto de forma (denominación) y contenido (significado), lo que cabe preguntarnos es cómo es la relación entre los conceptos y los términos a la vista de que una misma categoría conceptual puede proyectarse en distintos términos, cada uno de los cuales puede transmitir un sentido distinto. Estos términos son grosso modo sinónimos, pero no en su totalidad, ya que a través de la denominación nos hacen percibir una parte o una faceta distinta del mismo concepto que representan.

En el trasfondo de esta digresión, si asumimos la concepción de la unidad terminológica como signo compuesto de forma y contenido, se esconde una cuestión clave para explicarnos la naturaleza de la unidad terminológica y su papel en todo el proceso de construcción y verbalización del conocimiento: mientras que la denominación se entiende claramente como la unidad formal que verbaliza el concepto, no es tan clara la relación que se da entre el significado y el concepto. ¿Se trata de una misma noción o de nociones diferentes? ¿Son lo mismo el significado y el concepto?

Un hecho que a lo largo de los últimos decenios ha sido subrayado por muchísimos autores ha sido la falta de evidencia empírica de la biunivocidad terminológica propuesta por E. Wüster. Cierto es que Wüster no hizo una aproximación descriptiva a la terminología, o si la hizo, como hemos mencionado en otras publicaciones (CABRÉ 2005) tomó como corpus de datos el conjunto de términos normalizados contenidos en su obra terminográfica. La consecuencia de ello es que Wüster trabajó sobre una terminología ideal, en lugar de trabajar sobre la terminología real, con lo la idea de que para afianzar al máximo la comunicación técnica había que tender a usar o tomar como referencia una única denominación para representar un solo concepto bien delimitado dentro de un ámbito temático. Esta situación ideal basada en la descripción de datos previamente normalizados le llevó a formular el principio de la biunivocidad del término, tan ampliamente criticado desde la terminología descriptiva, aunque justificado en el contexto empírico en el que Wüster desarrolló su teoría.

El principio de la biunivocidad, además de no ajustarse a la realidad de los datos representativos del uso terminológico, se apoya en la relación directa entre el concepto y su denominación. Si se acepta esta relación directa y se asume que el concepto y el término son 
productos uniformizados mediante un proceso de normalización, no cabe preguntarse qué relación existe entre residuos y deshechos porque en realidad se tratará de dos denominaciones alternativas de un mismo concepto. La pregunta es si se trata de un mismo concepto, o bien si se trata de dos unidades terminológicas distintas, y si cada una de ellas representa una asociación concepto-denominación distinta. Lo cierto en cualquier caso es que estamos ante un fenómeno de variación, lo que no vemos tan claro es si se trata de un caso de variación terminológica global o de variación denominativa, es decir puramente formal.

A la vista de los ejemplos que hemos comentado y partiendo de la concepción del término como signo, creemos que es posible hipotetizar sobre tres puntos:

- la estructura compleja del concepto

- la variación en la proyección del concepto en el término

- el carácter motivado de la denominación del término

La concepción del concepto como una estructura compleja ya ha sido desarrollada dentro de la teoría del prototipo. Esta teoría sin embargo deja fuera de la explicación la multidimensionalidad del concepto, en el sentido de que las características que lo describen pueden agruparse en conjuntos, cada uno de los cuales puede representar una faceta del mismo y a menudo cada faceta puede estar determinada por un criterio o dimensión. Autores como Pustejowsky (1995) han desarrollado una teoría del significado léxico como una estructura de estructuras, que fácilmente puede acoplarse a nuestra propuesta (KUGUEL, 2007), propuesta que puede sintetizarse en los siguientes puntos:

- el concepto es una estructura compleja, plural en cuanto a características y facetada en cuanto a sus dimensiones;

- el concepto puede proyectarse globalmente en un término, pero también puede proyectarse en distintos términos y resultar todos ellos sinónimos;

- el concepto puede proyectarse en uno o más términos no motivados, pero puede proyectarse también en distintos términos motivados, que presentan entre si variación formal y pueden tener sentidos distintos, ya que la proyección de cada faceta de un concepto en un término comporta un cambio de sentido;

- la ocurrencia de un término en un enunciado puede entenderse como una "instanciación" del concepto;

- la variación denominativa se repara en el discurso; la variación denominativa con consecuencias cognitivas puede expresarse a través de la forma denominativa en sí o por pistas discursivas que actúan de indicios de variación conceptual;

- estos indicios pueden ser de muchos tipos y pueden expresarse mediante distintas formas gramaticales. ${ }^{10}$

\section{VOLVIENDO A LOS OBJETIVOS Y A MODO DE CONCLUSIÓN}

Hemos dicho al inicio de este texto que nuestro objetivo era doble:

Por un lado, nos proponíamos argumentar sobre la necesidad de postular distintos planos dentro de la terminología para poder explicar adecuadamente tanto la compleja articulación del proceso realidad-concepto-término, como determinados fenómenos que conciernen a más de un plano.

Por otro lado, queríamos mostrar algunas de las correlaciones existentes entre los distintos planos, correlaciones que en este artículo hemos centrado en la compleja relación entre un concepto y su múltiple terminologización ${ }^{11}$, y dentro de este caso nos hemos interesado más específicamente por la variación denominativa con consecuencias cognitivas.

\footnotetext{
${ }^{10}$ Un caso sería el uso de adverbios o frases adverbiales para explicitar las distintas dimensiones de un mismo concepto: La enfermedad $X$, desde el punto de visto anatomopatológico, se describe como...; desde el punto de vista sintomático, se describe como...”.

${ }^{11}$ Usamos politerminologización, formado a partir del término polidenominación de Freixa 2002, para indicar cuando un concepto se realiza a través de más de un término y estos términos presentan entre sí una sinonimia parcial por cuanto actualizan distintos sentidos, todos ellos a partir de proyecciones de distintas facetas de un mismo concepto.
} 
Hemos analizado las distintas fases del proceso de terminologización, desde la aprehensión de la realidad en forma de clases de objetos a través de filtros que condicionan esta aprehensión, pasando por la categorización de cada clase de objetos en forma de concepto y su ubicación en la mente integrando virtualmente una o más estructuras de conceptos, hasta llegar a la verbalización del concepto a través del término.

Para ello hemos distinguido tres planos de análisis de la terminología:

- el plano referencial, que comprende el ámbito de los objetos y de la formación de clases de objetos

- el plano cognitivo, que incluye la formación de conceptos y su ubicación en la mente

- el plano lingüístico, o más ampliamente semiótico si integramos los signos de naturaleza artificial en la denominación "término", que comprende la realización de cada concepto en una o más unidades terminológicas del lenguaje natural, que pueden ampliarse a otras unidades de creación artificial.

Hemos visto que la relación entre los tres planos no es simple, sino compleja y diversa, de forma que a una clase de objetos categorizada en un concepto pueden corresponderle una o más unidades terminológicas, concebidas como signos, es decir, unidades de forma y contenido.

Hemos visto también que dentro un mismo campo de especialidad pueden existir distintas unidades terminológicas correspondientes a conceptos totalmente diferentes o bien correspondientes total o parcialmente a un mismo concepto. En los casos de correspondencia total, hablamos de sinonimia a secas: la variación denominativa se produce en la forma, pero no causa distorsión en el significado. En los casos de correspondencia parcial hablamos de sinonimia parcial, por cuanto el signo se asocia a un mismo concepto, pero no existe variación únicamente en la denominación sino también en el contenido que expresa. Es en este segundo caso en que la denominación está motivada y remite a un concepto sin actualizarlo completamente ni globalmente, sino solo de manera parcial, frecuentemente tomando como criterio la actualización de una de las facetas del concepto.

Para poder dar cuenta de esta compleja relación entre un concepto y sus diferentes realizaciones terminológicas hemos postulado que es necesario partir de distintos planos de análisis, contrariamente nunca podríamos explicar como sinónimos casos de sinonimia parcial cuya denominación está motivada, es decir, es en ella misma un indicio del sentido que se actualiza en cada término.

Con este análisis pensamos que podemos formular más ampliamente el principio de la poliedricidad que hasta hoy habíamos aplicado sólo a las unidades terminológicas según el cual los términos son unidades interdisciplinarias integradas por vertientes o facetas distintas, cada una de ellas correspondiente a un plano de análisis. Con este artículo hemos intentado mostrar que el principio de poliedricidad puede aplicarse también al concepto, por cuanto es desde su carácter multifacético o poliédrico que podemos explicar los casos de variación denominativa con consecuencias cognitivas. Estos casos no son más que proyecciones en el plano lingüístico de la poliedricidad conceptual.

La distinción de planos de análisis, aparte de resultar metodológicamente potente, es coherente con el postulado del carácter interdisciplinario de la terminología y de la posibilidad de abordarla desde diferentes ópticas, como ha sido representado a través del modelo de las puertas (CABRÉ, 2000; 2003).

\section{BIBLIOGRAFÍA}

ADELSTEIN, A. Unidad léxica y significado especializado: modelo de representación a partir del nombre relacional "madre". Tesis doctoral. Barcelona: Institut Universitari de Linguística Aplicada, 2007.

CABRÉ, M. T. "Terminologie et linguistique: la théorie des portes". Terminologies nouvelles: Terminologie et diversité culturelle. Broussels, n. 21, p.10-15, 2000.

" "Theories of terminology. Their description, prescription and explanation". Terminology, Amsterdam, v. 9, n.2, p. 163-200, 2003. 
— . "La Terminologia, una disciplina en evolución: pasado, presente y algunos elementos de futuro". Debate Terminológico. v. 1. n.1, 2005. Disponível em http://riterm.net/revista/ojs/index.php/debateterminologico/article/view/23/45 Arquivo acessado em 28/04/2011.

http://www.riterm.net/revista/n_1/index.htmFREIXA, J. La variació terminològica: anàlisi de la variació denominativa en textos de diferent grau d'especialització de l'àrea de medi ambient. Tesis doctoral. Barcelona: Institut Universitari de Lingüística Aplicada, Universitat Pompeu Fabra, 2002

INTERNATIONAL ORGANIZATION FOR STANDARDIZATION ISO 1087 -1 Terminology work -- Vocabulary -- Part 1: Theory and application. Genebre: ISO, 1997.

. ISO 1087-1: Terminology work -- Vocabulary -- Part 1: Theory and application. Genebre: ISO, 2000.

. ISO 1087-2: Terminology work -- Vocabulary Part 2: Computer applications. Genebre: ISO, 1997

. ISO 1087-2: Terminology work -- Vocabulary Part 2: Computer applications. 2000

KUGUEL, I. La semántica del léxico especializado: los términos en textos de ecología. Tesis doctoral. Buenos Aires: Facultad de Filosofía y Letras, Universidad de Buenos Aires, 2007.

LAKOFF, G. Women, fire, and dangerous things: what categories reveal about the mind. Chicago: University of Chicago Press, 1987.

LEECH, G. N. Semantics: The Study of Meaning. $2^{a}$ edición. London: Penguin, 1990.

PUSTEJOVSKY, J. The Generative lexicon. Cambridge. Mass: MIT Press, 1995.

RONDEAU, G. Introduction à la terminologie. Boucherville, Québec: Gaëtan Morin, 1984.

ROSCH, E. "Principles of categorization". In: __ Lloyd, B. B. (ed.). Cognition and Categorization. Hillsdale (NJ): Lawrence Erlbaum Associates, 1978. p. 27-48.

TEBÉ, C. La representació conceptual en terminologia: l'atribució temàtica en els bancs de dades terminològiques. Tesis doctoral. Barcelona: Institut Universitari de Lingüística Aplicada, Universitat Pompeu Fabra, 2005

TEMMERMAN, R. Towards New Ways of Terminology Description: The SociocognitiveApproach. Amsterdam: John Benjamins, 2000.

VANGEHUCHTEN, L. El Léxico del discurso económico empresarial identificación, selección y enseñanza en español como lengua extranjera con fines específicos. Madrid, Frankfurt and Main: Iberoamericana, Vervuert, 2005.

WÜSTER, E. Einführung in die Allgemeneine Terminologielehre und Terminolische Lexicographie. Viena: Springer. 1979. Versión española: CABRÉ, M. T. (dir.) Introducción a la teoría de la terminología y a la lexicografía terminológica. Barcelona: Institut Universitari de Lingüística Aplicada, Universitat Pompeu Fabra, 1998.

\footnotetext{
*** En Freixa (2002) se analiza la variación denominativa y se presenta una tipología de casos muy amplia, según la cual existen variantes denominativas de cuatro tipos: a) basadas en cambios gráficos, b) basadas en cambios morfosintácticos, c) basadas en reducciones, d) basadas en cambios léxicos, y e) basadas en varios cambios complejos. Reproduzcamos a continuación dicho gráfico:
}

\begin{tabular}{|c|c|c|}
\hline & Tipus & Subtipus \\
\hline \multirow[t]{6}{*}{ I. Canvis gràfics } & \multirow[t]{3}{*}{ 1. Terme i forma artificial } & a) Terme i símbol \\
\hline & & b) Terme i fórmula química \\
\hline & & c) Terme $\mathrm{i}$ altra forma artificial \\
\hline & \multirow[t]{2}{*}{ 2. Terme i abreviació } & d) Terme i sigla \\
\hline & & e) Terme i abreviatura \\
\hline & 3. Canvi ortogràfic & \\
\hline \multirow{4}{*}{$\begin{array}{l}\text { II. Canvis } \\
\text { morfosintàctics }\end{array}$} & \multirow[t]{4}{*}{ 1. Manteniment de l'estructura } & a) Absència / presència d'article \\
\hline & & b) Canvi de nombre \\
\hline & & c) Canvi de preposició \\
\hline & & d) Canvi de gènere \\
\hline
\end{tabular}




\begin{tabular}{|c|c|c|}
\hline & \multirow[t]{3}{*}{ 2. Canvi de l'estructura } & e) $[\mathrm{N}+\mathrm{A}] /[\mathrm{N}+\mathrm{SP}]$ \\
\hline & & f) Monolèxic / polilèxic \\
\hline & & g) Altres canvis d'estructura \\
\hline \multirow[t]{7}{*}{ III. Reduccions } & \multirow[t]{3}{*}{ 1. Reduccions de l'extensió } & $\begin{array}{ll}\text { a) } & \text { Tipus }[\mathrm{N}+\mathrm{X}]=[\mathrm{N}] \\
: & {[\mathrm{N}+\mathrm{A}]=[\mathrm{N}]} \\
: & {[\mathrm{N}+\mathrm{SP}]=[\mathrm{N}]} \\
& {\left[\mathrm{N}_{1}+\mathrm{N}_{2}\right]=\left[\mathrm{N}_{1}\right]}\end{array}$ \\
\hline & & 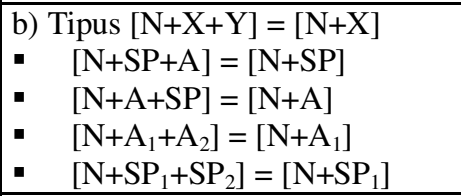 \\
\hline & & c) Altres reduccions de l'extensió \\
\hline & \multirow{3}{*}{ 2. Reduccions de la base } & d) $[\mathrm{N}+\mathrm{A}]=[\mathrm{A}]_{\mathrm{N}}$ \\
\hline & & e) $\left[\mathrm{N}_{1}+\mathrm{N}_{2}\right]=\left[\mathrm{N}_{2}\right]$ \\
\hline & & f) $\left[\mathrm{N}_{1}+\mathrm{SP}\left(d e+\mathrm{N}_{2}\right)\right]=\left[\mathrm{N}_{2}\right]$ \\
\hline & 3. Altres reduccions & \\
\hline \multirow[t]{3}{*}{ IV. Canvis lèxics } & 1. Unitats monolèxiques & \\
\hline & \multirow[t]{2}{*}{ 2. Unitats polilèxiques } & $\begin{aligned} & \text { a) Canvi de base } \\
& \text { : } {[\mathrm{N}+\mathrm{SP}(\text { prep }+ \text { art }+\mathrm{N})] } \\
& \text { - } {[\mathrm{N}+\mathrm{A}] } \\
& \text { altres canvis de base }\end{aligned}$ \\
\hline & & $\begin{aligned} & \text { b) Canvi d'extensió } \\
& \text { - } {[\mathrm{N}+\mathrm{SP}(\text { pre }+ \text { art }+\mathrm{N})] } \\
& \text { - } {[\mathrm{N}+\mathrm{A}] } \\
& \text { - } \text { altres canvis d'extensió }\end{aligned}$ \\
\hline \multirow{4}{*}{$\begin{array}{l}\text { V. Diversos canvis } \\
\text { complexos }\end{array}$} & \multirow[t]{2}{*}{ 1. Amb parentiu formal } & a) monolèxic / polilèxic \\
\hline & & b) polilèxic / polilèxic \\
\hline & \multirow[t]{2}{*}{ 2. Sense parentiu formal } & c) monolèxic / polilèxic \\
\hline & & d) polilèxic / polilèxic \\
\hline
\end{tabular}

Figura extraída de Freixa (2002: 283-284).

Los tipos establecidos por Freixa están basados en la observación de las denominaciones, pero de todas ellas, para poder formular una hipótesis sobre la relación término concepto, los únicos casos que nos interesan son solo aquellos que presentan variación denominativa y esta variación afecta a la relación de la denominación con el concepto, o dicho de otro modo, nos interesa saber qué tipos de variación denominativa pueden tener consecuencias cognitivas y cuáles son estas consecuencias. 